\title{
NUMERICAL INVESTIGATION OF PRESSURE ACTING ON FLOATING PANEL FOR WAVE OVERTOPPING REDUCTION UNDER REGULAR WAVE ACTION
}

\author{
Koji Kawasaki ${ }^{1}$, Han Dinh $\mathrm{Ut}^{2}$, Tetsuya Matsuno ${ }^{3}$ and Tadashi Fukumoto ${ }^{4}$
}

\begin{abstract}
In this paper, a 3-D multiphase flow model with solid-gas-liquid interaction, named 'DOLPHIN-3D', is utilized to numerically investigate the characteristics of pressure acting on a floating panel, which is installed in front of an upright seawall for wave overtopping reduction. The validity and utility of the model were confirmed through good agreements between the numerical results and experimental ones in terms of the dynamic response of the floating panel and the pressure at the bottom of the panel. The numerical results revealed that the model can appropriately simulate the pressure acting on the floating panel as well as the dynamic behavior of the panel under wave action.
\end{abstract}

Keywords: Multiphase flow model; solid-gas-liquid interaction; pressure; floating panel; wave overtopping reduction

\section{INTRODUCTION}

It is acknowledged that coastal zones are at risk of huge coastal disasters caused by tsunami, storm surge, extreme wave, wave overtopping and so on. To prevent the coastal zones from the abovementioned disasters, a lot of countermeasures such as vertical seawalls, wave absorbing breakwaters and submerged breakwaters has so far been constructed in coastal sea areas. Although these structures have certainly contributed to the safety of civilians and the development at the coastal areas, the disaster prevention functions of these existing structures are currently required to be improved to adapt with global climate change in terms of the increasing severity of storm events and sea level rise related to disaster such as storm surge and wave overtopping, as well as the social demand concerned with water environment and cost reduction of public works.

In a previous paper (Kawasaki et al. 2011a), an adaptive countermeasure to prevent and mitigate wave overtopping disasters induced by storm surge or high wave was proposed, in which a floating panel was newly installed to the front of an existing upright seawall. The function of the floating panel for a wave overtopping countermeasure was furthermore examined by conducting laboratory experiments. Great effectiveness of the floating panel, which can reduce wave overtopping by following water surface elevation in front of the seawall, was then confirmed. The practical application of floating panel as a wave overtopping reduction countermeasure is therefore promising. However, in order to fully obtain data for technical design stage, not only laboratory experiments but numerical investigations are needed. Kawasaki et al. (2011b) utilized a multiphase flow model with solid-gasliquid interactions "DOLPHIN-3D" (Dynamic numerical model Of muLti-Phase flow with Hydrodynamic INteraction-3 Dimension version), which was proposed by Kawasaki and Ogiso (2009), to numerically investigate dynamic behavior of the floating panel under regular wave action. It was found from this study that the multiphase flow model is capable of reproducing the dynamic behavior of the floating panel under wave action. Dynamic response characteristics of the floating panel to the incident wave period and wave pressure acting on the floating panel, which are the most important factors for the design stage of the floating panel, nonetheless, have not been sufficiently investigated yet.

In this paper, the multiphase flow model "DOLPHIN-3D" is used to investigate the response characteristics of the floating panel to the incident wave period. Moreover, the numerical investigations on wave pressure acting on the floating panel are discussed.

The paper is organized as follow. Firstly, laboratory experiment is briefly introduced. Next, the multiphase flow model "DOLPHIN-3D" are addressed. Numerical results and discussions are then presented. The paper ends with some conclusions.

\footnotetext{
${ }^{1}$ Associate professor, Department of Civil Engineering, Nagoya University, C1-3(651), Furo-cho, Chikusa-ku, Nagoya 464-8603, Japan

${ }^{2}$ Engineer, Corporate Strategy Department, Central Nippon Highway Engineering Nagoya Co., Ltd., 1-8-11 Nishiki, Naka-ku, Nagoya 464-0003, Japan

${ }^{3}$ Master Student, Department of Civil Engineering, Nagoya University, C1-3(651), Furo-cho, Chikusa-ku, Nagoya 464-8603, Japan

${ }^{4}$ Engineer, Nishimatsu Construction Co., Ltd., 20-10, 1-chome, Toranomon, Minato-ku, Tokyo 105-8401, Japan
} 


\section{LABORATORY EXPERIMENT}

Hydraulic model experiments were perform in a two-dimensional wave flume $(30 \mathrm{~m}$ long, $0.9 \mathrm{~m}$ high and $0.7 \mathrm{~m}$ wide) at Coastal and Ocean Engineering Laboratory, Nagoya University, as shown in Fig. 1. The scale ratio between model and prototype was set to $1 / 20$. The wave flume has a piston-type wave generator at one side, whereas a wave absorber is located at the other side. The system for wave overtopping reduction is presented in Fig. 2 . In the system, a floating panel $(0.2 \mathrm{~m}$ high, $0.03 \mathrm{~m}$ thick and $0.66 \mathrm{~m}$ wide) with a $0.1 \mathrm{~m}$ draft was installed to the front of an upright seawall with a $0.525 \mathrm{~m}$ high, which was located at $22.72 \mathrm{~m}$ far from the wave generator. The horizontal movement of the floating panel was constrained by a steel frame, which was connected to the seawall. The variation of the floating panel motion, the water surface elevation and wave overtopping phenomena around the panel were captured with a digital high-speed camera HAS-D3. The images were then analyzed to obtain numerical data of floating panel motion by employing an image processing program named DIPPMotion Pro (Ditect Co., Ltd.). Wave pressure sensor PS-05KC was installed at the bottom of the floating panel to measure pressure acting on the panel, as indicated in Fig.3.

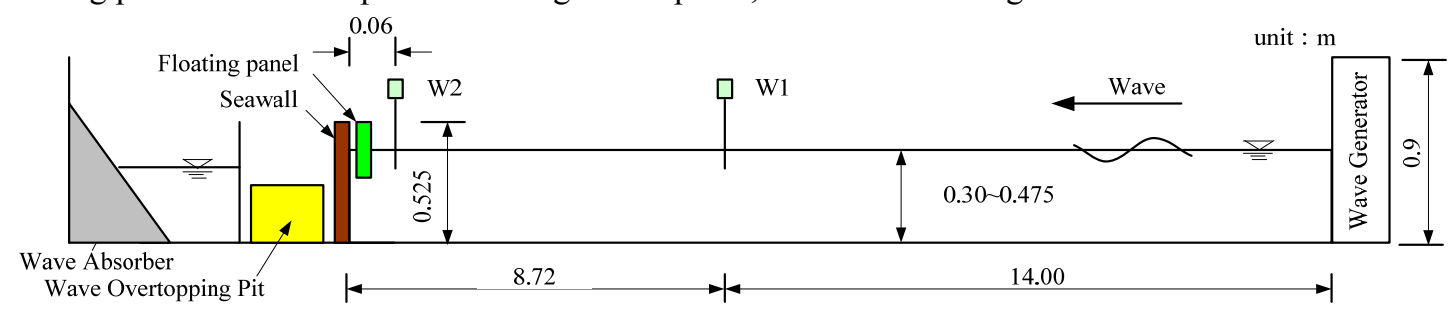

Figure 1. Experiment setup

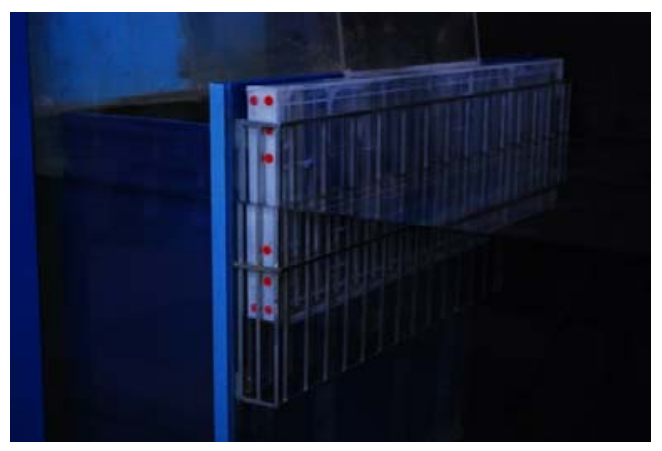

Figure 2. Wave overtopping reduction system

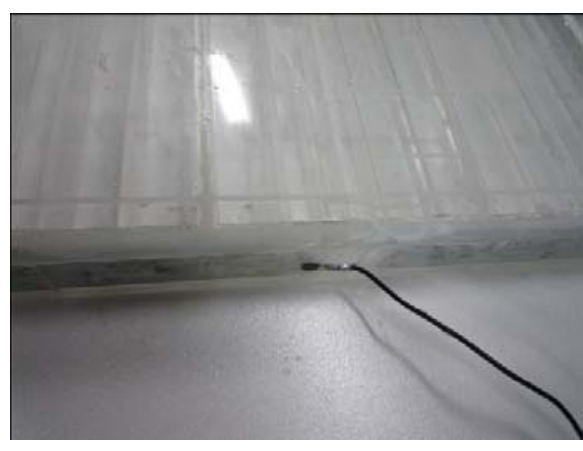

Figure 3. Wave pressure sensor installation

\section{3-D MULTIPHASE FLOW MODEL WITH SOLID-GAS-LIQUID INTERACTION}

\section{Governing Equations}

The governing equations consist of the conservation equation of mass (Eq. 1), the Navier-Stokes equations (Eq. 2), the pressure equation for compressible fluid (Eq. 3), the advection equation of density functions (Eq. 4) and the equation of state for barotropic fluid (Eq. 5). The equations allow computing precisely not only incompressible but also compressible multiphase flows.

$$
\begin{aligned}
& \frac{\partial \rho}{\partial t}+\frac{\partial\left(u_{j} \rho\right)}{\partial x_{j}}=\rho q \\
& \frac{\partial u_{i}}{\partial t}+u_{j} \frac{\partial u_{i}}{\partial x_{j}}=-\frac{1}{\rho} \frac{\partial p}{\partial x_{i}}-g_{i}+\frac{f_{s i}}{\rho}+\frac{\partial}{\partial x_{j}}\left(-\tau_{i j}+2 \frac{\mu}{\rho} S_{i j}\right)-D_{i} u_{i} \\
& \frac{\partial p}{\partial t}+u_{j} \frac{\partial p}{\partial x_{j}}=-\rho C_{l s}^{2}\left(\frac{\partial u_{j}}{\partial x_{j}}-q\right) \\
& \frac{\partial \phi_{I}}{\partial t}+u_{j} \frac{\partial \phi_{I}}{\partial x_{j}}=0
\end{aligned}
$$




$$
\begin{aligned}
& \rho=f(p) \\
& q=\left\{\begin{array}{cc}
0 & \left(x \neq x_{s}\right) \\
q^{*} & \left(x=x_{s}\right)
\end{array}\right.
\end{aligned}
$$

where, $x_{i}$ is position vector $(x, y, z), u_{i}$ is velocity component in the direction of $i, \rho$ is fluid density, $p$ is pressure, $g_{i}$ is gravitational acceleration vector $(0,0,-g), f_{s i}$ is surface tension term, $\tau_{i j}$ is turbulence term, $\mu$ is the coefficient of viscosity, $S_{i j}$ is strain rate tensor $\left(\partial u_{i} / \partial x_{j}+\partial u_{j} / \partial x_{i}\right), D_{i}$ is dissipation coefficient used in energy dissipation zones, $C_{l s}$ is local sound speed, $t$ is time and $\phi_{I}(I=1 \sim 3)$ are density functions for respective phases ( $\phi_{1}$ : solid phase; $\phi_{2}$ : liquid phase; $\phi_{3}$ : gas phase) that represent the rate of fractional volume for each phase in a cell and these functions need to satisfy the relationship: $\phi_{1}+\phi_{2}+\phi_{3}=1\left(0 \leq \phi_{I} \leq 1\right)$ in a cell. $q=q(z, t)$ is wave generation source with its strength $q^{*}$ assigned only at source line $\left(x=x_{s}\right)$.

\section{Computational Algorithm}

Fig. 4 indicates the computational flow chart of the multiphase flow model. Eqs. 2 and 3 are divided into an advection step and a non-advection step by making use of a time splitting method as shown in Eqs. $7 \sim 10$. The resultant equations are discretized by employing irregular staggered mesh grids. A CIP method developed by Yabe and Aoki (1991) is used to calculate the hyperbolic equations for all variables at the advection step, while equations at the non-advection step are solved with an extended SMAC method (Kawasaki, 2005), which can simulate both compressible and incompressible fluid. Eq. 1 is solved by a CIP-CSL2 method proposed by Nakamura et al. (2001), which is one of the conservative methods extended from a CIP method. The effect of surface tension on the gas-liquid interface is evaluated by using a CSF (Continuum Surface Force) model proposed by Brackbill et al. (1992), which interprets surface tension as a continuous mass force across the interface. A LES (Large Eddy Simulation) -based on a DTM (Dynamic Two-parameter Mixed) model developed by Salvetti et al. (1995) is applied for estimating turbulence quantities.

[Advection step]

$$
\begin{aligned}
& \frac{\partial u_{i}}{\partial t}+u_{j} \frac{\partial u_{i}}{\partial x_{j}}=0 \\
& \frac{\partial p}{\partial t}+u_{j} \frac{\partial p}{\partial x_{j}}=0
\end{aligned}
$$

[Non-advection step]

$$
\begin{aligned}
\frac{\partial u_{i}^{n+1}}{\partial t} & =-\frac{1}{\rho^{n}} \frac{\partial p^{n+1}}{\partial x_{i}}+F_{i} \\
\frac{\partial p^{n+1}}{\partial t} & =-\rho C_{l s}^{2}\left(\frac{\partial u_{j}^{n+1}}{\partial x_{j}}-q\right)
\end{aligned}
$$

where, $F_{i}$ represents external force term such as gravity, viscous, surface tension and dissipation zone terms.

\section{Numerical procedure at advection step}

The equations at the advection step are calculated by using the CIP method with 3rd-order accuracy, which can solve the advection equation precisely, taking advantage of the hyperbolic equation $f$ as represented in Eq. 11.

$$
\frac{\partial f}{\partial t}+u_{j} \frac{\partial f}{\partial x_{j}}=0
$$




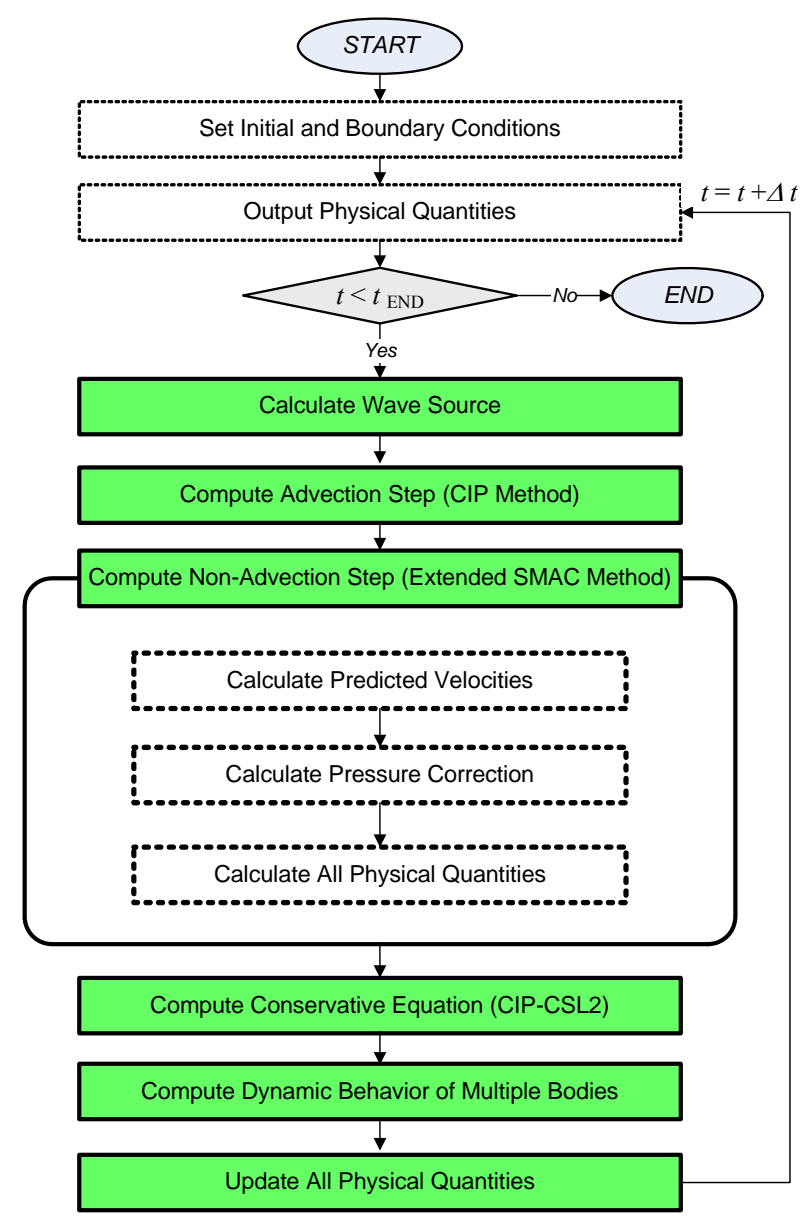

Figure 4. Flow chart of multiphase flow model

Fig. 5 shows the concept of one-dimensional CIP method, in which the spatial distribution of value is interpolated in the range of the interval $\left[x_{i-1}, x_{i}\right]$ with a cubic polynomial function formed as Eq. 12 .

$$
F_{i}^{n}(x)=a_{1} X^{3}+a_{2} X^{2}+a_{3} X+a_{4}
$$

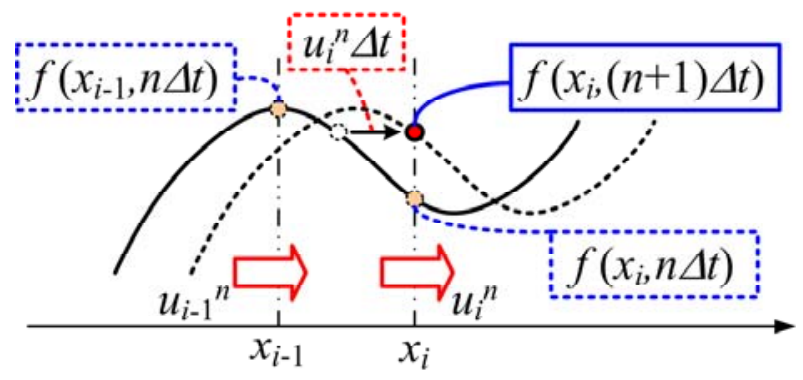

Figure.5. Concept of one-dimensional CIP method

The unknown coefficient $a_{1} \sim a_{4}$ in the interpolation function of $F_{i}^{n}(x)$ are determined from the continuities of $f$ and its spatial derivatives $f_{x}$ at the grid points $x_{i-1}$ and $x_{i}$. Then, value $f_{i}^{*}$ at the next time step is computed by Eq. 13.

$$
f_{i}^{*}=F_{i}^{n}\left(x_{i}-u_{i} \Delta t\right)
$$




\section{Numerical procedure at non-advection step}

Eqs. 9 and 10 cannot be solved explicitly since the unknown variables at the next time step are included in both the left and the right sides of the equations. In the model, the extended SMAC method is used to compute both compressible and incompressible fluids.

The predicted velocity $\tilde{u}_{i}$ is computed explicitly by Eq. 14 with the help of variables after the advection step.

$$
\frac{\widetilde{u}_{i}-u_{i}^{*}}{\Delta t}=-\frac{1}{\rho} \nabla p^{*}+F_{i}^{*}
$$

where, superscript * represents the time step after the advection step.

The simultaneous equation for the pressure correction $\delta p=p^{n+1}-p^{*}$ in Eq. 15 is derived by eliminating $\nabla \cdot u_{i}^{n+1}$ from Eq. 10 using Eqs. 9 and 14.

$$
\nabla\left(\frac{1}{\rho^{*}} \nabla \delta p\right)=\left(\frac{\delta p}{\rho^{*} C_{l s}^{2} \Delta t^{2}}-q\right)+\frac{1}{\Delta t} \frac{\partial \widetilde{u}_{i}}{\partial x_{i}}
$$

The pressure correction $\delta p$ in Eq. 15 is solved by an ILUCGS (Incomplete LU decomposition Conjugate Gradient Squared) method. Finally, all the variables at the next time step $t=(n+1) \Delta t$ are updated by Eqs. $16 \sim 18$.

$$
\begin{aligned}
& u_{i}^{n+1}=\tilde{u}_{i}-\frac{\Delta t}{\rho^{*}} \nabla \delta p \\
& p_{i}^{n+1}=p_{i}^{*}+\delta p \\
& \rho^{n+1}=\rho^{*}-\rho^{*} \nabla \cdot u_{i}^{n+1} \Delta t
\end{aligned}
$$

The local sound speed $C_{l s}$ and the viscous coefficient $\mu$ for each cell are evaluated from Eqs. 19 and 20 .

$$
\begin{aligned}
& C_{l s}=\phi_{1} C_{l s 1}+\phi_{2} C_{l s 2}+\phi_{3} C_{l s 3} \\
& \mu=\phi_{1} \mu_{1}+\phi_{2} \mu_{2}+\phi_{3} \mu_{3}
\end{aligned}
$$

where, the subscripts 1, 2 and 3 represent solid, liquid and gas phases, respectively.

\section{Motion Equation of Floating Panel}

In the laboratory experiment, it was seen that the motion of the floating panel under wave action sometimes induces a collision between the steel frame and floating panel. Due to the collision, the impact of the friction force between the floating panel and the steel frame was observed when the floating panel moved in the vertical direction. This force is assumed to influence the dynamic behavior of the floating panel in the vertical direction. The motion equation of the floating panel is, therefore, proposed by adding a damping term to the equation of the motion in the vertical direction. On the other hand, it was found from the laboratory experiment that the vertical motion of the floating panel was dominant compared with rotational and horizontal motions. Two latter motions are therefore neglected in this study.

Under the above-mentioned assumption, the motion equation of the floating panel is denoted as follows.

$$
M_{l} \frac{d w_{l}^{n+1}}{d t}+c w_{l}^{n+1}=F^{*}
$$

where, $M_{l}$ is the total mass of the floating panel, $c$ is a damping coefficient, $F^{*}$ is the hydrodynamic force term at the present step, and $w_{l}^{n+1}$ and $d w_{l}^{n+1} / d t$ represent the velocity and the acceleration of the gravity center of the floating panel motion at the next time step, respectively. 
In order to solve the equation of motion, the Newmark- $\beta$ method is utilized to determine the relation of the velocity at the next time step $w_{l}{ }^{n+1}$ to the velocity at the present time step $w_{l}^{n}$ as follows,

$$
w_{l}^{n+1}=w_{l}^{n}+\left(\frac{d w_{l}^{n}}{d t}+\frac{d w_{l}^{n+1}}{d t}\right) \frac{\Delta t}{2}
$$

By substituting $w_{l}^{n+1}$ in Eq. 22 into Eq. 21, the acceleration at next time step is obtained in Eq.23,

$$
\frac{d w_{l}^{n+1}}{d t}=\frac{-c\left(w_{l}^{n}+\frac{d w_{l}^{n}}{d t} \frac{\Delta t}{2}\right)+F^{*}}{M_{l}+c \frac{\Delta t}{2}}
$$

\section{NUMERICAL RESULTS AND DISCUSSION}

\section{Computational Conditions}

Numerical calculations were carried out under regular wave conditions with wave height $H=0.04 \mathrm{~m}$ and $0.10 \mathrm{~m}$ and wave period $T=0.75 \mathrm{~s}, 1.0 \mathrm{~s}$ and $2.24 \mathrm{~s}$. A definition sketch of a computational domain used in numerical simulations for a still water depth $h=0.425 \mathrm{~m}$ is depicted in Fig. 6 . The computational domain includes an energy dissipation zone on the left and a wave overtopping pit on the right. The origin of $x$ coincides with the wave generation source, and the positive direction of $x$ is taken toward the right hand side of the computational domain. The location of the wave generation source was set near the left side of the analysis domain. A wave gauge W2 was installed in front of the seawall in order to measure water surface elevation. Mesh sizes $\Delta x / L_{i}$ and $\Delta z / h$ ( $L$ : wavelength) in the respective direction of $x$ and $z$ are varied in a range of 1/200 1/100 and 1/40 1/25. The mesh sizes in the possible motion space of the floating panel were set equal to $0.005 \mathrm{~m}$ in order to capture the surface of the floating panel with high accuracy. The time interval at every time step was set at $0.0001 \mathrm{~s}$ so that the Courant condition is always satisfied.

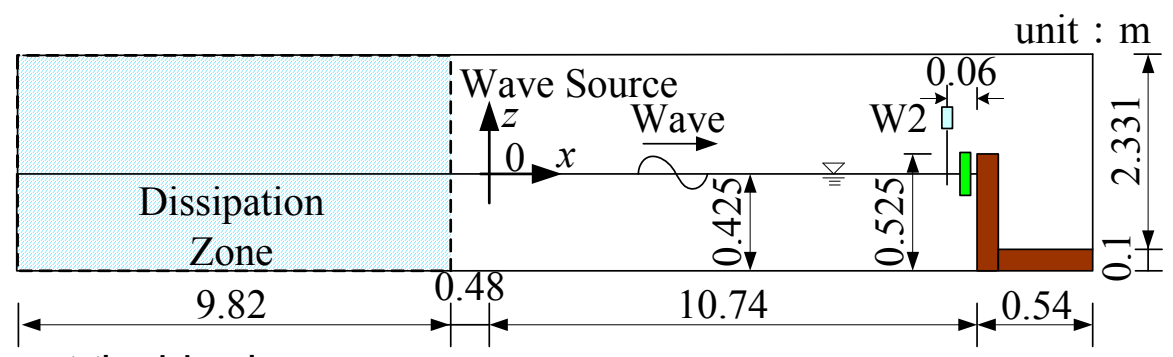

Figure 6. Computational domain

\section{Dynamic Response of Floating Panel under Wave Action}

The validity of the numerical model in terms of the dynamic behavior of the floating panel was confirmed in Kawasaki et al. (2011b). Hereafter, the relation of dynamic response of the floating panel to incident wave period is numerically discussed.

Figures 7 and 8 show the temporal variation of floating panel and water surface elevation for the numerical results and experimental ones, respectively. The continuous and dashed lines represent the floating panel motion and the water surface elevation. (a) and (b) in Figs. 7 and 8 indicate the incident wave condition of $H=0.04 \mathrm{~m}$, whereas Fig. (c) is the case of $H=0.10 \mathrm{~m}$. It is recognized from these figures that the phase lag between the water surface elevation and the floating panel motion becomes larger when the period of incident wave is close to natural period of floating panel $T_{s}=0.74 \mathrm{~s}$. On the other hand, the amplitude of the floating panel motion is found to be decreased. For the same wave period of $T=1.0 \mathrm{~s}$, the vertical motion of floating panel for the condition of wave height $H=0.1 \mathrm{~m}$ is larger than that for the condition of $H=0.04 \mathrm{~m}$ because of non-linearity of wave. The validity of the numerical model for the dynamic response of floating panel and water surface elevation is furthermore verified through the comparison between Figs. 7 and 8. 


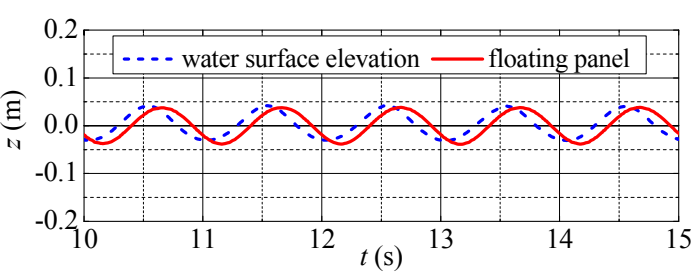

(a) $H=0.04 \mathrm{~m}, T=1.0 \mathrm{~s}$

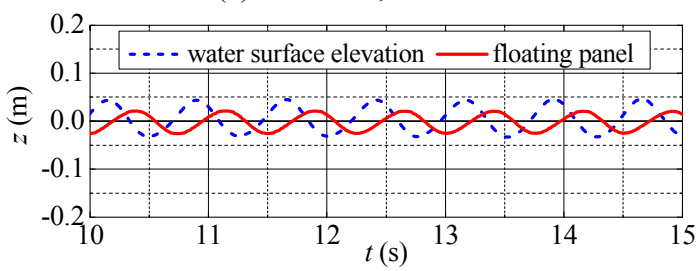

(b) $H=0.04 \mathrm{~m}, T=0.75 \mathrm{~s}$

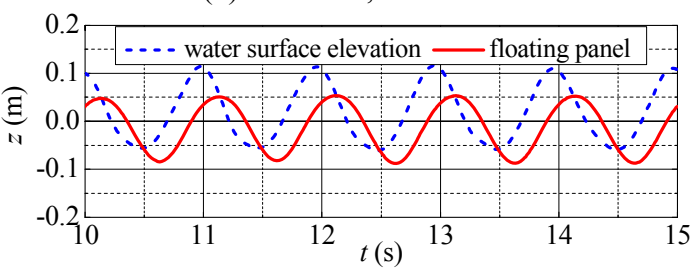

(c) $H=0.10 \mathrm{~m}, T=1.0 \mathrm{~s}$

Figure 7. Temporal variation of water surface elevation and floating panel motion under wave action (numerical results)

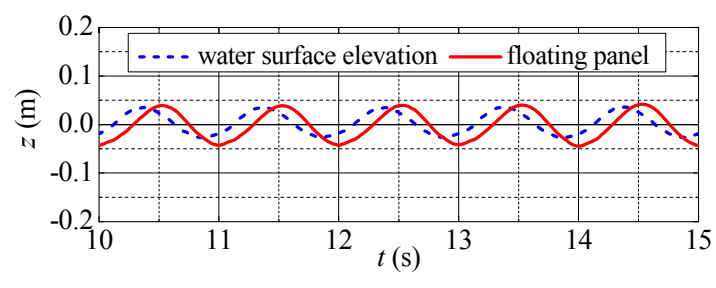

(a) $H=0.04 \mathrm{~m}, T=1.0 \mathrm{~s}$

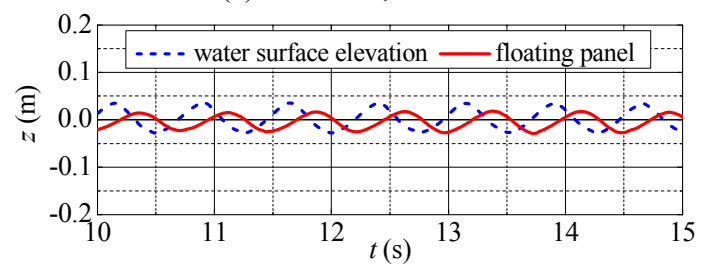

(b) $H=0.04 \mathrm{~m}, T=0.75 \mathrm{~s}$

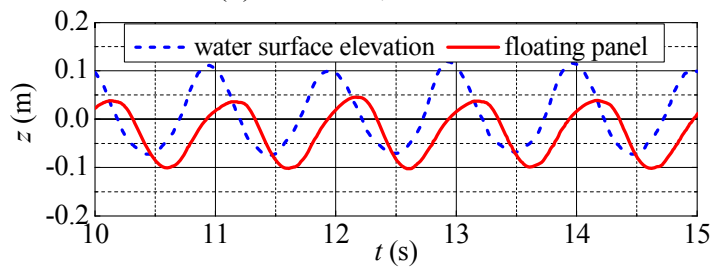

(c) $H=0.10 \mathrm{~m}, T=1.0 \mathrm{~s}$

Figure 8. Temporal variation of water surface elevation and floating panel motion under wave action (experimental results)

Next, a POV-Ray (Persistence Of Vision Raytracer), which is a high quality ray tracing program for creating three-dimensional graphics in the form of realistic reflection, refraction and light, was used to draw wave field around the floating panel and floating panel. Figures 9(a) and (b) show the hydraulic phenomena around the floating panel for the condition of same wave height $H=0.10 \mathrm{~m}$ and wave period $T=2.24 \mathrm{~s}$ and $T=1.0 \mathrm{~s}$, respectively. As seen from these figures, for the condition of wave period of $T=2.24 \mathrm{~s}$, the water surface elevation well follows the floating panel so the wave overtopping from the crest of the seawall is effectively prevented. On the other hand, when the wave period of $T=1.0 \mathrm{~s}$, which is close to the natural period of the floating panel motion $T=0.74 \mathrm{~s}$, water mass overtops the crest of the seawall because of the clear delay in phase between floating panel motion and water surface elevation. Therefore, in order to discuss the wave overtopping reduction effect of the floating panel, it is necessary to understand the influence of the relation of natural period of floating panel motion and the wave period on the floating panel motion.

Figures 10 and 11 show the relation of the normalized period ratio of wave period and the natural period of floating panel motion to the normalized amplitude and phase lag, respectively. The normalized amplitude is defined here as the ratio of the amplitude of floating panel motion $A_{s}$ and the amplitude of the water surface elevation in front of the seawall $A_{w f}$. Good agreements between the calculated and measured results seen in both Figs. 10 and 11 confirm the validity of the numerical model. Furthermore, it is found from Fig.10 that if the ratio of the natural period of floating panel motion and wave period $T_{s} / T$ is small, the normalized amplitude is close to 1 , meaning that the amplitude of floating panel motion is approximately the amplitude of the water surface elevation in front of the panel. When $T_{s} / T$ is close to 1 , the normalized amplitude is found to be slightly larger than 1. Also, the normalized amplitude decreases gradually when $T_{s} / T$ is larger than 1 . On the other hand, it is seen from Fig. 11 that when the normalized period ratio increases, the phase lag decreases rapidly. Consequently, the natural period of the floating panel motion is found to influence not only the amplitude of floating panel motion but also the phase lag, which are significant factors for wave overtopping reduction performance. 

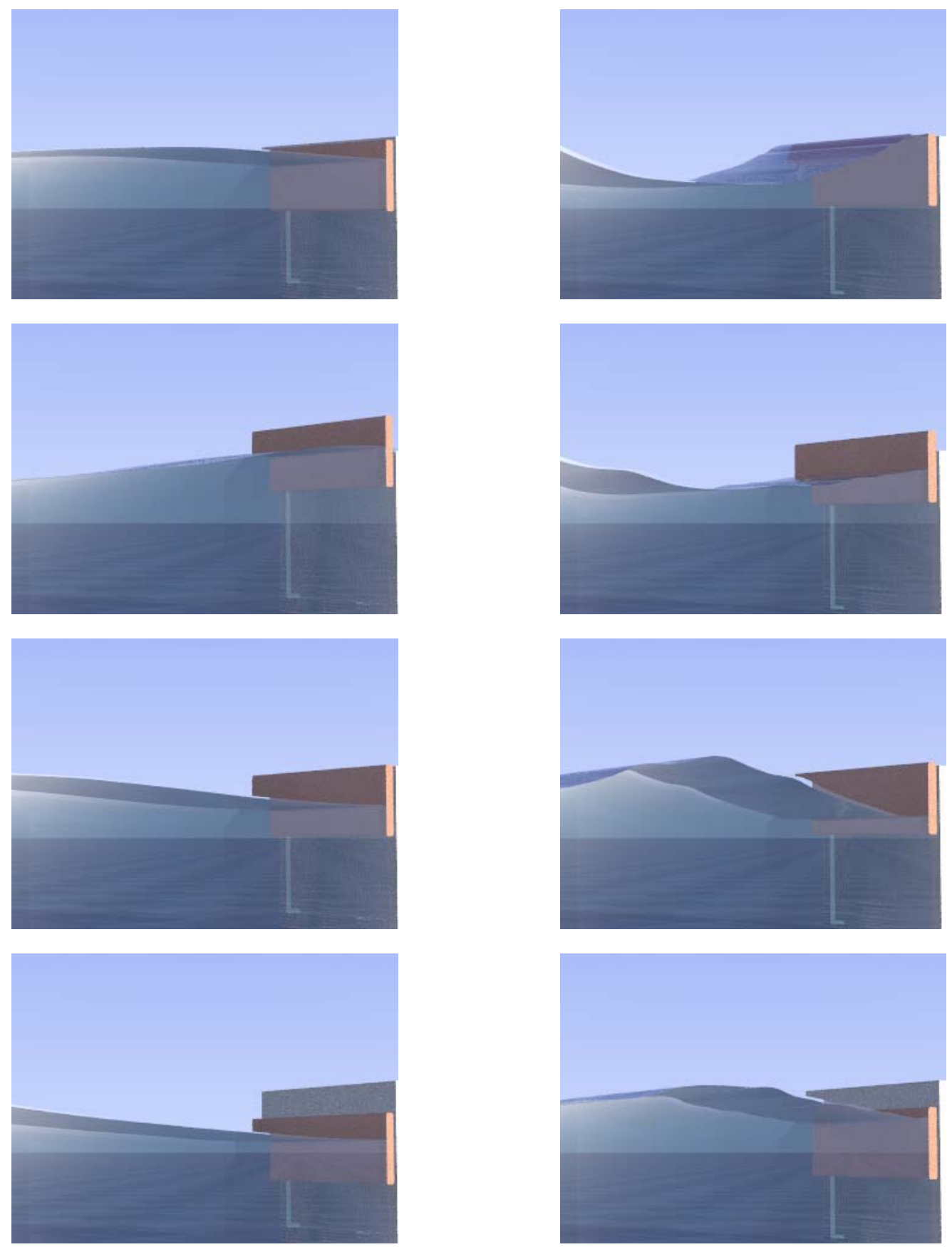

(a) $H=0.10 \mathrm{~m}, T=2.24 \mathrm{~s}$

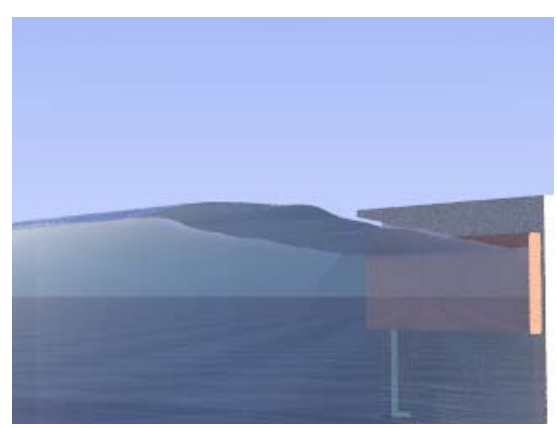

(b) $H=0.10 \mathrm{~m}, T=1.0 \mathrm{~s}$

Figure 9. Three dimensional view of hydraulic phenomena around floating panel with POV-Ray

\section{Pressure Acting on the Floating Panel}

Figure 12 shows the temporal variation of pressure acting on the bottom of the floating panel, in which the solid lines and circle symbols indicate the numerical results and experimental ones, respectively. It should be noted that the pressure in Fig. 12 represents dynamic pressure under wave action, namely difference from the hydrostatic pressure. It is found from Figs. 12(a) (c) that the calculated results are in good agreement with measured ones. The numerical model is, therefore found to be capable of reproducing wave pressure acting on the floating panel. The model is a useful tool to compensate the difficulties in measuring wave pressure acting on the floating panel in laboratory experiments. 
Figures 13 and 14 depict the temporal variation of the distribution of pressure acting on the floating panel under the wave condition of wave height $H=0.10 \mathrm{~m}$ and wave period of $T=2.24 \mathrm{~s}$ and $1.00 \mathrm{~s}$, respectively. In these figures, the bold line represents the distribution of hydrostatic pressure. From Fig. 13 , the distribution of wave pressure is found to be similar to that of the hydrostatic pressure. On the other hand, for wave period $T=1.00$ s shown in Fig. 14, the different between the distribution of wave pressure and that of hydrostatic pressure is clearly recognized, and this difference is, especially, seen to be largest at $t=13.00 \mathrm{~s}$. As mentioned before, when wave period is close to the natural period of floating panel motion $T_{s}=0.74 \mathrm{~s}$, the impact of collision and friction between floating panel and steel frame was seen to be larger. It would be said that noted large impact results in the large difference in terms of speed between floating panel motion and water surface elevation, meaning that the large difference between wave pressure and water surface elevation occurs.

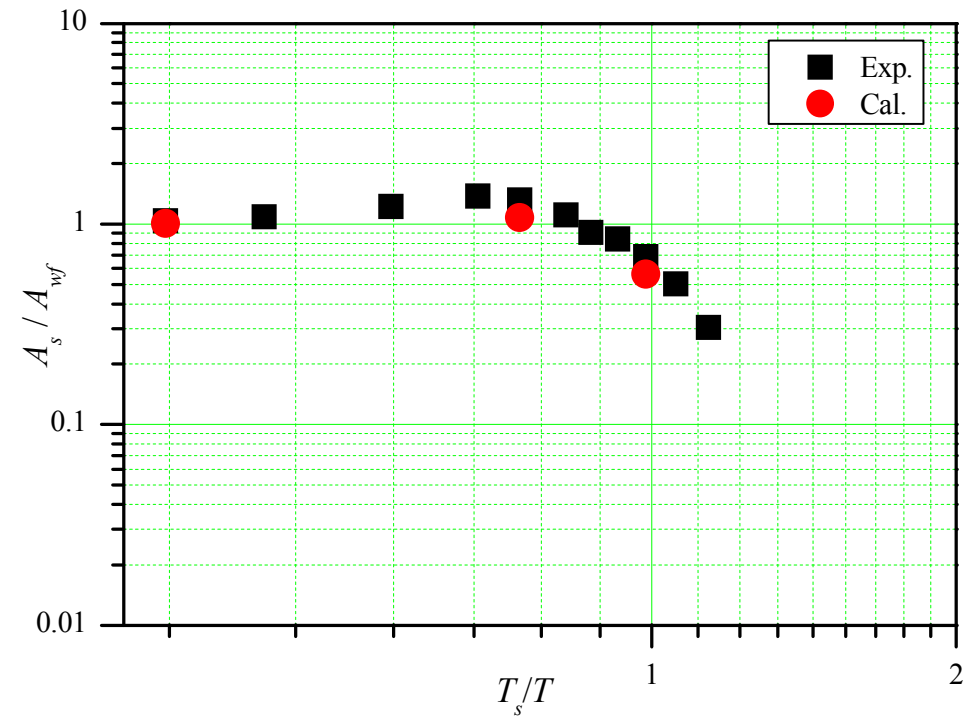

Figure 10. The relation of the normalized amplitude $A_{s} / A_{s w}$ to $T_{s} / T$

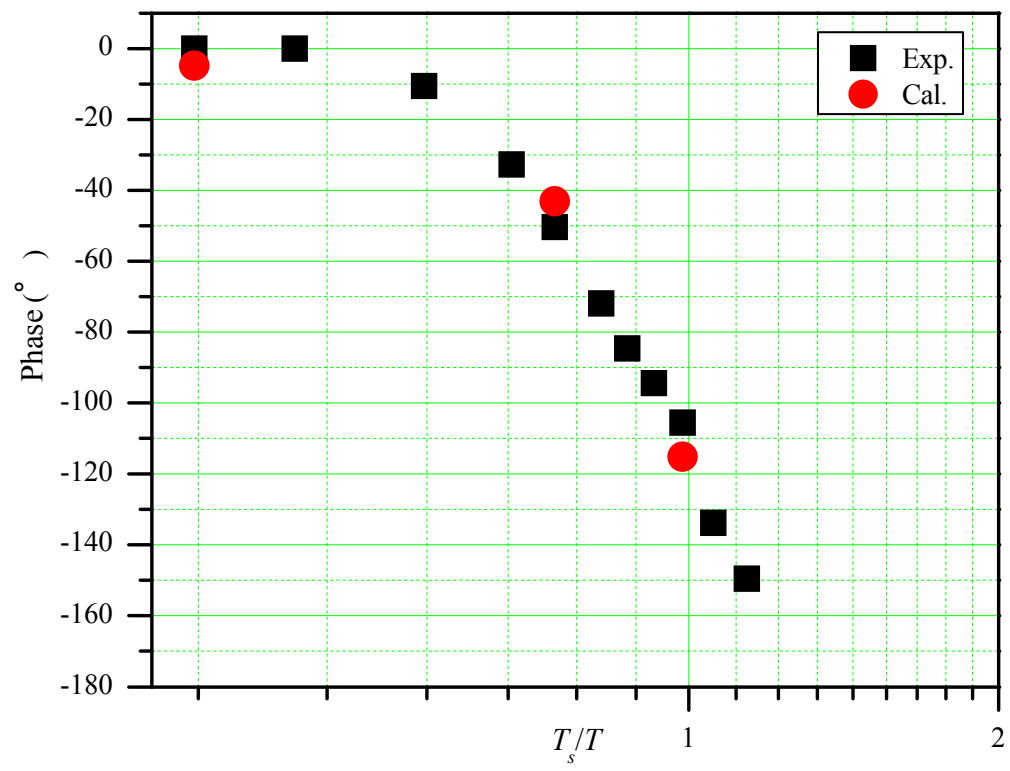

Figure 11. Relation of the phase $\operatorname{lag} A_{s} / A_{s w}$ to $T_{s} / T$ 


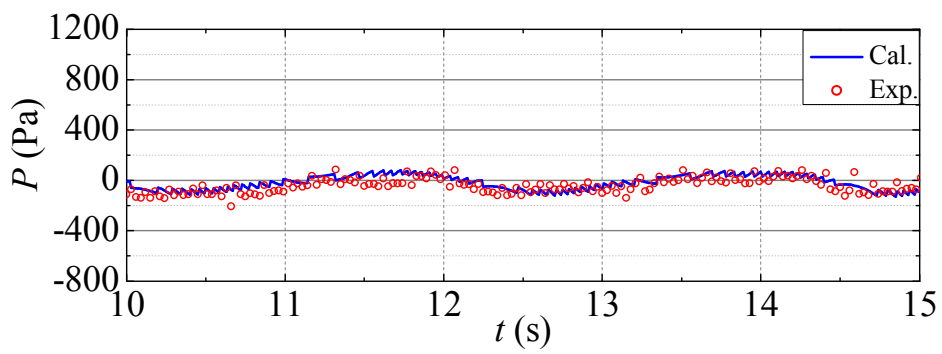

(a) $H=0.04 \mathrm{~m}, T=2.24 \mathrm{~s}$

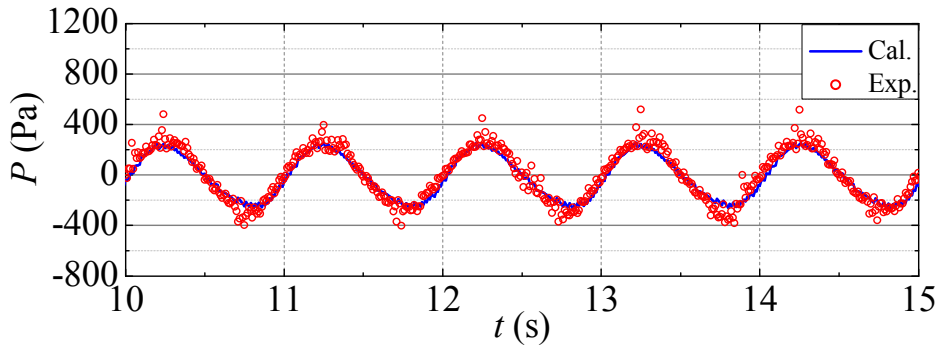

(b) $H=0.04 \mathrm{~m}, T=1.0 \mathrm{~s}$

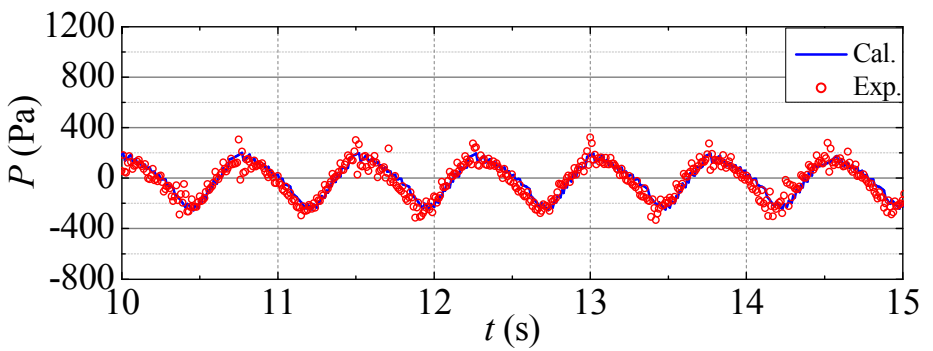

(c) $H=0.04 \mathrm{~m}, T=0.75 \mathrm{~s}$

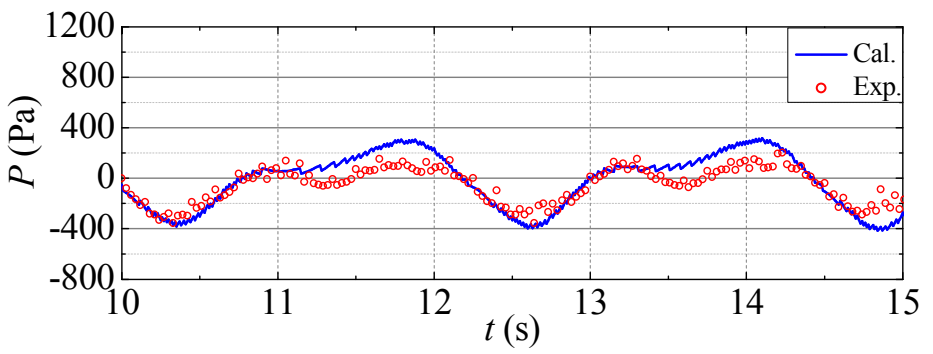

(d) $H=0.10 \mathrm{~m}, T=2.24 \mathrm{~s}$

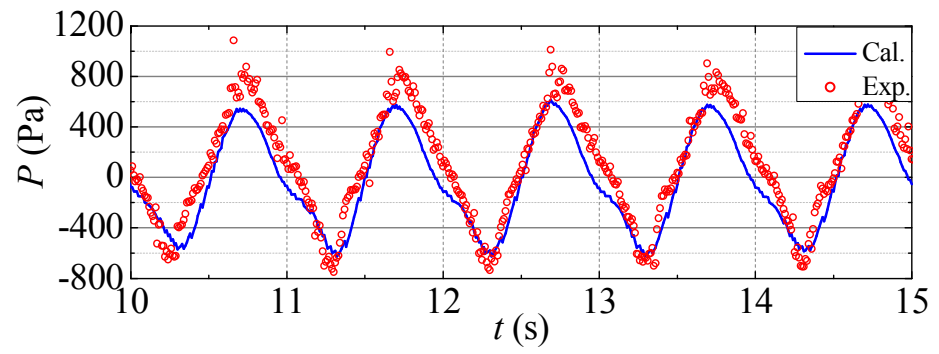

(e) $H=0.10 \mathrm{~m}, T=1.0 \mathrm{~s}$

Figure 12. Time variation of pressure acting on the bottom of floating panel

\section{CONCLUSION}

A multiphase flow model with solid-gas-liquid interaction "DOLPHIN-3D" was utilized to investigate pressure acting on a floating panel motion for wave overtopping reduction, which was installed to the front of an existing seawall. Good agreements between numerical and experimental results confirmed the validity and the utility of the numerical model. The validity of the model was furthermore verified through analyzing pressure acting on the front and back sides of the floating panel 
under wave action, which is one of the most important factors in designing coastal structures. The measurement of pressure exerting on a movable structure is in general difficult in laboratory experiment, and the numerical model, therefore, would be said to be a very helpful analysis tool in design of coastal structures.
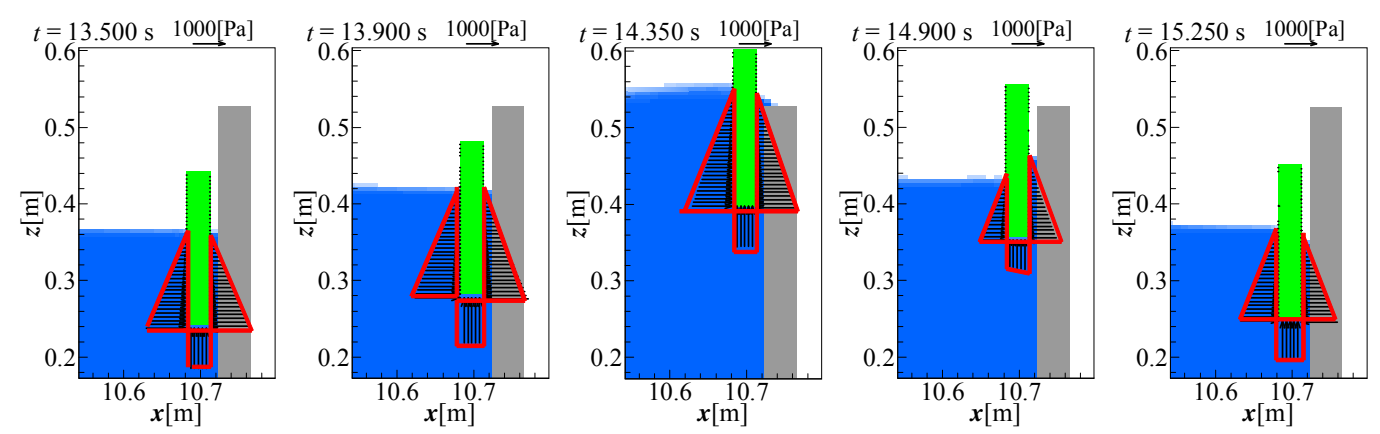

Figure 13. The distribution of pressure acting on the floating panel $(H=0.1 \mathrm{~m}, T=2.24 \mathrm{~s})$
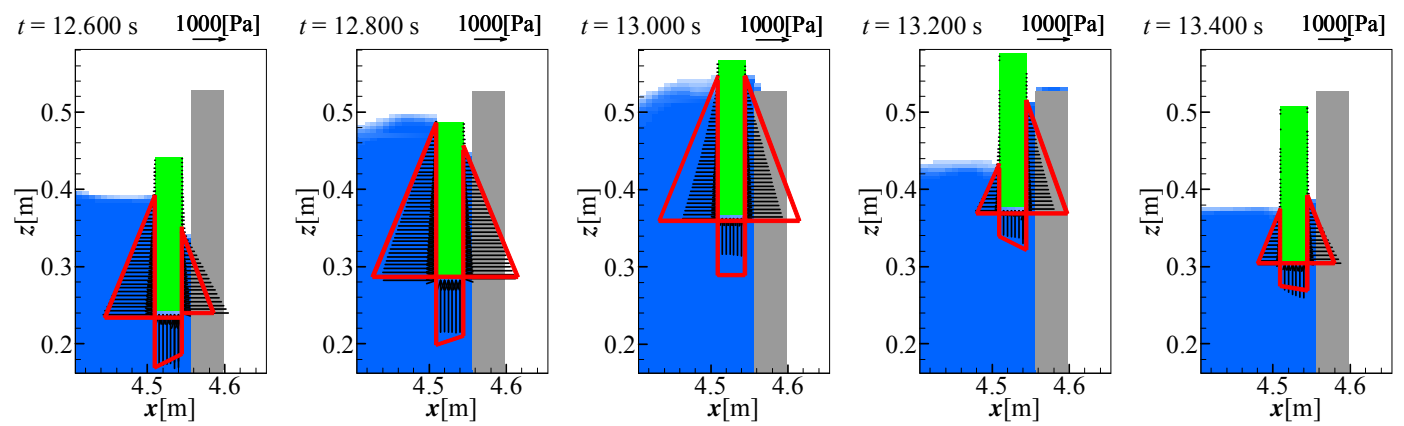

Figure 14. The distribution of pressure acting on the floating panel $(H=0.1 \mathrm{~m}, T=1.0 \mathrm{~s})$

\section{REFERENCES}

Brackbill, J. U., Kothe, D. B. and C. Zemach, 1992. A continuum method for modeling of surface tension, J. Computational Physics, 100: 335-354.

Kawasaki, K., Dinh Ut, H., Funahashi,T., T. Fukumoto, 2011a. Experimental Investigation on Adaptive Countermeasure Using Floating Panel for Wave Overtopping Reduction, The $21^{s t}$ Int. Offshore and Polar Engineering Conference, 1100-1107.

Kawasaki, K., Dinh Ut, H., Matsuno T., Funahashi,T., T. Fukumoto, 2011b. Multiphase flow simulation of dynamic behavior of floating panel for wave overtopping reduction under regular wave action, Proceeding of International Coastal Structures 2011, 12p (in press).

Kawasaki, K., and K. Ogiso. 2009. Development of 3-D multiphase flow numerical model "DOLPHIN-3D" and its application to wave-rigid body interaction problems, Proceedings of the 31st International Conference on Coastal Engineering 2008, 3199-3211.

Kawasaki, K., 2005. Numerical model of 2-D multiphase flow with solid-gas-liquid interaction, Int. Journal of Offshore and Polar Engineering, Vol.15, 3: 198-203.

Nakamura, T., Tanaka, R., Yabe, T., and K. Takizawa, 2001. Exactly conservative semi-Lagrangian scheme for multi-dimensional hyperbolic equations with directional splitting technique, $J$. Computational Physics, 174: 171-207.

Salvetti, M.V. and S. Banerjee, 1995. A priori tests of a new subgrid-scale model for finite-difference large-eddy simulations, Physic. of Fluids, 7: 2831-2847.

Yabe, T. and T. Aoki,1991.A universal solver for hyperbolic equations by cubic-polynomial interpolation I. One-dimensional solver, Comp. Phys. Communications, 66:219- 232. 